\title{
Síndrome da Hiperémese dos Canabinoides: Uma Revisão Prática para Clínicos
}

\section{Cannabinoid Hyperemesis Syndrome: A Practical Review for Clinicians}

Tiago Ferreira ${ }^{1}$, Catarina Oliveira ${ }^{1}$, Sara Dehanov ${ }^{1}$, Inês Figueiredo ${ }^{1}$, Manuel Dias ${ }^{1}$, Mariana Morins²

\section{RESUMO}

A primeira descrição clínica da síndrome da hiperémese dos canabinoides advém de um artigo de Allen et al publicado em 2004. Desde então, várias publicações têm-se focado na sua conceptualização e abordagem clínica, embora se mantenha pouco conhecida na comunidade médica. Esta síndrome é caracterizada pela tríade de consumo crónico de canabinoides, hiperémese cíclica, e banhos ou duches quentes compulsivos como forma de alívio sintomatológico. Podem ainda ocorrer dor abdominal e outros sintomas. A sua verdadeira prevalência é desconhecida, embora se acumule evidência de que é mais frequente do que outrora julgado. Foram aventadas várias hipóteses para a sua patogénese, nomeadamente relacionadas com recetores canabinoides e vaniloides, o eixo hipotálamo-hipófise-adrenal e o sistema nervoso simpático. Em termos terapêuticos, justificam-se medidas de suporte (p.e. hidratação) na fase aguda, existindo evidência para a utilização de benzodiazepinas (nomeadamente lorazepam), haloperidol ou capsaicina tópica para alívio sintomático. A única intervenção que interrompe o quadro é a abstinência dos consumos, devendo ser feita psicoeducação e orientação adequadas do doente. O conhecimento relativo a esta patologia encontra-se em crescimento. A falta de sensibilização para a doença pode conduzir a testes de diagnóstico invasivos e dispendiosos, inúmeras idas ao serviço de urgência e manutenção do quadro clínico sem resolução sintomatológica. O presente artigo visa sistematizar as linhas de intervenção clínica e fomentar uma maior consciencialização dos profissionais de saúde sobre esta entidade, permitindo uma melhor identificação e abordagem terapêutica deste quadro.

PALAVRAS-CHAVE: Abuso de Marijuana/complicações; Canabinoides/envenenamento; Comportamento Compulsivo/induzido quimicamente; Síndrome; Vómitos/induzido quimicamente; Vómitos/tratamento 


\section{ABSTRACT}

The first clinical description of cannabinoid hyperemesis syndrome comes from an article by Allen et al. published in 2004. Since then, several publications have focused on its conceptualization and clinical approach, although it remains largely unknown among the medical community. This syndrome is characterized by the triad of chronic cannabinoid consumption, cyclic hyperemesis, and compulsive hot baths or showers for symptom relief. Abdominal pain and other symptoms may also occur. Its true prevalence is unknown, despite growing evidence that it is more frequent than previously thought. There are several hypotheses that aim to explain its pathogenesis, namely related to the cannabinoid and vanilloid receptors, the hypothalamic-pituitary-adrenal axis and the sympathetic nervous system. Regarding its therapeutic approach, support measures could be justified in the acute phase (e.g. hydration), and there is evidence for the utilization of benzodiazepines (especially lorazepam), haloperidol and topical capsaicin for symptomatic relief. The only intervention that leads to the definite remission of symptoms is abstinence of cannabinoid use, with psychoeducation and adequate therapeutic guidance of the patient. Our knowledge about cannabinoid hyperemesis syndrome is still growing. The lack of awareness of the disease can lead to invasive and expensive diagnostic tests, multiple visits to the emergency department, and persistence of symptoms. This article aims to systematize the lines of clinical intervention of the disease and to promote a greater awareness of this entity among health professionals, allowing for a better identification and therapeutic approach of the condition.

KEYWORDS: Cannabinoids/poisoning; Compulsive Behavior/chemically induced; Marijuana Abuse/complications; Syndrome; Vomiting/chemically induced; Vomiting/therapy

\section{INTRODUÇÃO}

De acordo com as Nações Unidas, a canábis é a droga recreativa mais consumida em todo o mundo com aproximadamente 192 milhões de consumidores estimados em 2016. ${ }^{1}$ O enquadramento legal do uso de canábis tem vindo a alterar-se de forma rápida em países como os Estados Unidos e o Canadá, com a aprovação do seu uso medicinal ou recreativo, fomentando a discussão deste tópico em outros países como Portugal. Tal situação, tem levado a um aumento do número de consumidores, e consequente incremento do aparecimento de efeitos secundários ligados a esta substância. ${ }^{2}$ Uma destas consequências, a síndrome da hiperémese dos canabinoides (SHC), tem sido reconhecida com frequência crescente na literatura nas últimas duas décadas, apesar de ainda aparentar ser desconhecida pela comunidade médica, nomeadamente na prática clínica. ${ }^{3}$

A primeira descrição clínica do quadro advém duma série de casos por Allen et al (2004) de nove pacientes australianos, aos quais acrescia um caso previamente reportado por More et al (1996). Verificou-se um padrão cíclico de episódios de vómitos precedido por anos de consumos de canabinoides, com resolução do quadro após cessação dos consumos, e alívio destes sintomas com banhos quentes na fase aguda. ${ }^{4}$ Baseados no trabalho de Allen et al, vários relatos e séries de casos começaram a aparecer. Em 2009, Sontineti et al aprofundaram a descrição das características clínicas da SHC e propuseram um conjunto de critérios de diagnóstico. ${ }^{5}$ Mais tarde, em 2012, Simonetto et al viriam a publicar outra série de casos ( $n=98)$ e a propor um conjunto mais alargado de critérios clínicos diagnósticos para a $\mathrm{SHC}^{6}$ (Tabela 1). Considera-se atualmente que a SHC é reconhecida pela tríade de consumo crónico de canabinoides, hiperémese cíclica, e banhos ou duches quentes compulsivos como forma de alívio sintomatológico?

Apesar destas tentativas de clarificação nosológica e de ter sido incorporada nos critérios Roma IV, ${ }^{8}$ esta síndrome não está reconhecida na ICD-10. ${ }^{9}$ A sua patogenia ainda não se encontra esclarecida, e existem poucos estudos de maior escala relacionados com as opções terapêuticas. Sendo uma perturbação que pode ter um elevado impacto para o doente e para os sistemas de saúde, é necessária maior divulgação da sua orientação diagnóstica e terapêutica. Assim, e motivados pela crescente observação de casos de SHC em serviço de urgência (SU), o nosso objetivo consistiu em realizar uma revisão sobre esta patologia com o intuito de difundir conhecimento sobre a mesma e de oferecer um artigo com a sistematização das linhas diagnósticas e de intervenção para clínicos.

\section{METODOLOGIA}

Revisão não-sistemática por pesquisa de artigos em base de dados PubMed pela palavra-chave "Cannabinoid Hyperemesis Syndrome" publicados entre 2004 e fevereiro de 2019. A pesquisa inicial devolveu 147 artigos, dos quais foram excluídos 101 após leitura do abstract por 
não se adequarem aos objetivos propostos. Utilizámos assim 46 artigos que nos permitiram descrever as manifestações clínicas, diagnóstico, epidemiologia, etiopatogenia e complicações da SHC, além da evidência atual relativa às melhores opções terapêuticas.

\section{RESULTADOS \\ MANIFESTAÇÕES CLÍNICAS}

Clinicamente, a SHC caracteriza-se por episódios recorrentes (cíclicos) de náuseas intensas e vómitos, usualmente de conteúdo aquoso, acompanhados por dor abdominal, epigástrica ou periumbilical. Podem ainda ocorrer sintomas psico-vegetativos (rubor facial ou diaforese). Estas manifestações geralmente apresentam predomínio matinal. São ainda importantes para o diagnóstico a presença de alívio sintomático com banhos ou duches quentes e existir relação obrigatória com o consumo crónico e habitualmente diário de canábis. $\bigcirc$ quadro parece surgir entre 1 a 5 anos após o início dos consumos, verificando-se alguma variabilidade, com relatos de casos de início entre os 4 meses a 27 anos. ${ }^{6,10}$ $\bigcirc$ período inter-episódico caracteriza-se pelo retorno ao estado de bem-estar, embora possam persistir dispepsia e vómitos ocasionais ou dor abdominal. A maioria dos indivíduos apresenta sete ou mais episódio por ano, experienciando o retorno dos sintomas com intervalos habitualmente inferiores a 2 meses. ${ }^{6}$

Podemos conceptualizar a evolução da SHC em três fases (Fig. 1):

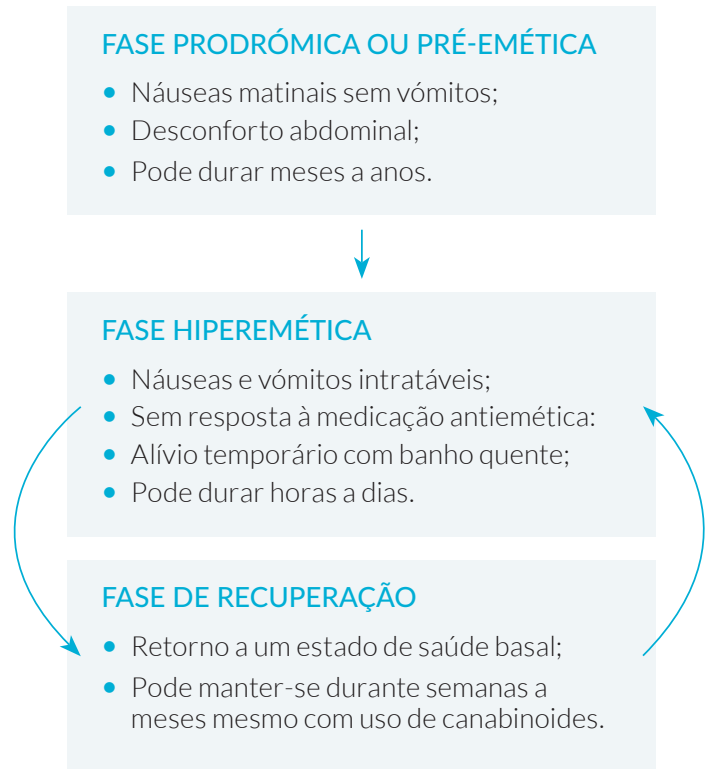

FIGURA 1. Esquema ilustrativo das fases da SHC. Adaptado de Fleming JE, et al. Cannabinoid Hyperemesis Syndrome. Fed Pract. 2017;34):33-6.?
- Prodrómica ou pré-emética: caracteriza-se por náuseas matinais sem vómitos e desconforto abdominal, com manutenção do padrão alimentar habitual..,11

- Hiperemética: fase mais sintomática, verificando-se náuseas e vómitos, que habitualmente não respondem à medicação antiemética. A intensidade dos vómitos é descrita como debilitante, com desenvolvimento de aversão à alimentação. É nesta fase que os pacientes procuram o banho quente como forma de alívio temporário dos sintomas, sendo também quando tendem a recorrer aos serviços médicos como o SU.7,11,12

- Recuperação: ocorre retorno ao estado basal, com normalização do padrão alimentar e da frequência dos banhos. A ausência de consumos leva a que esta fase se mantenha indefinidamente embora a recorrência de consumo leve ao reaparecimento da SHC.,11

\section{DIAGNÓSTICO}

Esta entidade é frequentemente subvalorizada, de baixa suspeição clínica e associada a atraso diagnóstico significativo (média 15,2 \pm 10,5 meses) com internamentos e investigações frequentemente inocentes e, muitas vezes, repetitivos. ${ }^{13}$ Simonetto et al propuseram critérios diagnósticos de SHC baseados em informações clínicas e em exames complementares diagnósticos sem alterações ${ }^{5,6}$ (Tabela 1).

Numa abordagem inicial, a história clínica e o exame físico dos indivíduos com náuseas, vómitos e dor abdominal devem ser dirigidos à exclusão de doenças potencialmente fatais. ${ }^{12}$ Após este passo o profissional de saúde deve questionar se existe melhoria sintomatológica com banhos quentes e qual a sua frequência e duração. Caso a resposta seja positiva deverá ser abordado o consumo

TABELA 1. Critérios de diagnóstico de SHC propostos por Simonetto et al, 2012. ${ }^{6}$

\section{Critérios Essenciais para o Diagnóstico de SHC}

Consumo crónico de canabinoides (diário).

Critérios Major para o Diagnóstico de SHC

Episódios cíclicos de náuseas intensas e vómitos; Resolução dos sintomas após cessação dos consumos; Alívio dos sintomas com banho quente;

Dor abdominal, epigástrica ou periumbilical;

Consumo semanal de canabinoides.

Critérios Sugestivos de SHC

Perda ponderal de 5 kg ou mais; Idade inferior a 50 anos; Predomínio matutino dos sintomas;

Manutenção dos hábitos intestinais normais;

Exames complementares sem alterações (laboratorial, endoscópico e radiográfico). 
de canabinoides, caracterizando a altura de início de consumo, a frequência e a quantidade. Deve-se caracterizar igualmente a data de início das náuseas e vómitos, dado que caso se verifique uma diferença de um ano ou mais entre o início de consumo crónico de canabinoides e as náuseas, é altamente provável que o diagnóstico seja de SHC. Por fim, a relação de melhoria das náuseas e vómitos com a cessação de canabinoides ou de recrudescimento com o retorno ao consumo é um elemento altamente sugestivo de SHC. ${ }^{6}$

\section{AVALIAÇÃO LABORATORIAL E IMAGIOLÓGICA}

A SHC associa-se, na maioria dos casos, a exames complementares de diagnóstico com resultados normais ou com alterações inespecíficas, devendo evitar-se uma investigações laboratorial e imagiológica extensas. ${ }^{11,12}$ Analiticamente o doseamento de canabinoides na urina deverá ser sempre pedido caso se verificarem os sintomas típicos de SHC, sem outra causa orgânica identificável, e perante a negação de consumos por parte do paciente. ${ }^{12}$ A restante avaliação laboratorial inicial deverá incluir o hemograma completo, glicose, função e enzimologia hepáticas, enzimas pancreáticas, função renal e parâmetros inflamatórios. 7,11,12

As opções de investigação imagiológica podem ser múltiplas, devendo ser adaptadas ao quadro clínico. Estas podem incluir o uso de radiografia abdominal, tomografia computorizada abdominal ou cranioencefálica, ecografia abdominal, endoscopia digestiva alta, colonoscopia, estudos de esvaziamento gástrico, cápsula endoscópica ou estudos das vias biliares. ${ }^{11}$ De salientar que os achados mais frequentes associados à SHC são o atraso do esvaziamento gástrico e a presença de padrão de gastrite leve ou inespecífica ou esofagite distal na endoscopia digestiva alta. ${ }^{7}$

\section{DIAGNÓSTICO DIFERENCIAL}

O diagnóstico diferencial de náuseas e vómitos cíclicos é muito vasto podendo incluir patologia do foro gastrointestinal ou peritoneal, endocrinológico, psiquiátrico, metabólico e do sistema nervoso central (SNC). ${ }^{7}$ Como referido, devem ser primeiramente excluídas as situações potencialmente fatais como, por exemplo, hepatite aguda, insuficiência adrenal, perfuração intestinal, obstrução intestinal, colangite, colecistite, diverticulite, gravidez etópica, gastroparésia, enfarte agudo do miocárdio, nefrolitíase, pancreatite, doença inflamatória pélvica ou rutura/dissecção de aneurisma da aorta. ${ }^{12}$

Outro diagnóstico frequentemente confundido com a SHC é a síndrome de vómitos cíclicos (SVC) (Tabela 2). Apesar do SVC apresentar sintomatologia semelhante à
TABELA 2. Diagnóstico diferencial de SVC e SHC. Adaptado de Ruffle JK, et al. Cannabinoid hyperemesis syndrome: an important differential diagnosis of persistent unexplained vomiting. Eur J Gastroenterol Hepatol. 2015;27(12):1403-8. ${ }^{13}$

\begin{tabular}{|c|c|c|}
\hline Características Clínicas & SVC & SHC \\
\hline Consumo de canabinoides & Pouco frequente & Necessário \\
\hline Distribuição por género & $F>M$ & $M>F$ \\
\hline Vómitos & Necessário & Necessário \\
\hline Dor abdominal & Frequente & Frequente \\
\hline Desidratação e polidipsia & Frequente & Frequente \\
\hline Perda de peso & Frequente & Frequente \\
\hline Banhos quentes frequentes & Ausente & Necessário \\
\hline Co-morbilidade psiquiátrica* & Frequente & Raro \\
\hline Co-morbilidade com enxaqueca & Frequente & Raro \\
\hline \multicolumn{3}{|c|}{ Exames Complementares de Diagnóstico } \\
\hline Leucocitose ou neutrofilia & Ocasional & Ocasional \\
\hline Hipocaliémia & Ocasional & Ocasional \\
\hline Esvaziamento gástrico & $\begin{array}{l}\text { Acelerado } \\
\text { a normal }\end{array}$ & $\begin{array}{l}\text { Atrasado } \\
\text { a normal }\end{array}$ \\
\hline $\begin{array}{l}\text { Doseamento de canabinoides } \\
\text { na urina }\end{array}$ & $\begin{array}{l}\text { Tipicamente } \\
\text { negativo }\end{array}$ & $\begin{array}{l}\text { Tipicamente } \\
\text { positivo }\end{array}$ \\
\hline Resultado endoscópico & Normal & $\begin{array}{l}\text { Esofagite } \\
\text { ou gastrite }\end{array}$ \\
\hline
\end{tabular}

*Depressão; Perturbação afetiva bipolar; Perturbação de ansiedade generalizada.

SHC, destacam-se aspetos da história clínica que aludem ao diagnóstico de SHC, nomeadamente a presença do comportamento aprendido de tomar banhos quentes repetidos para melhoria sintomática, assim como a história universalmente positiva de uso crónico de canábis. ${ }^{13}$

\section{EPIDEMIOLOGIA}

Os dados relativos à SHC baseiam-se predominantemente em relatos e séries de casos, não existindo dados fidedignos relativos à sua prevalência. Contudo, estima-se que a SHC não seja tão rara entre consumidores crónicos de canábis quanto se pensava. ${ }^{10}$ Num estudo de Aziz l et al (2018) relativo à prevalência das perturbações gastrointestinais funcionais com base nos critérios de Roma IV, a prevalência global da SHC foi de 0,1\% ( $n=7$ em 5931), correspondendo a 9,6\% dos casos de SVC ou 23,3\% dos casos de SVC com consumos de canábis. ${ }^{8}$ Num estudo por Habboushe J et al (2018) em que participaram 2127 doentes em contexto de SU, 32,9\% ( $n=51)$ dos 155 consumidores frequentes de canábis reuniam critérios para SHC. Ainda no mesmo estudo, não se encontraram diferenças demográficas entre os indivíduos com sintomas de SHC e aqueles sem sintomas. ${ }^{14}$ Outro estudo por Hernandez JM et al (2017) concluiu existência de subdiagnóstico desta entidade motivado 
pela baixa pesquisa ativa de historial de consumo de canábis, a confusão com outros diagnósticos como SVC, ou o facto do consumo ainda ser uma informação por vezes ocultada pelo próprio doente. ${ }^{15} \mathrm{Kim} \mathrm{HS}$ et al (2015), comprovaram que a prevalência de apresentações clínicas em urgência de vómitos cíclicos quase duplicou após a liberalização de canábis no estado do Colorado (EUA), ainda que mantendo um valor globalmente baixo, o que demonstraria que a SHC seria uma perturbação relativamente incomum, ainda que em crescendo. ${ }^{16} \mathrm{Um}$ estudo por Venkatesan T et al (2014) em que foi aplicado um questionário pela internet a doentes com SVC concluiu que a maioria destes (81\%) consumia canábis, sendo que o sexo masculino, banhos quentes e presença de patologia co-mórbida (como ansiedade e depressão) se associavam ao consumo de canábis. ${ }^{17}$

O surgimento dos primeiros sintomas ocorre habitualmente aos $22 \pm 5$ anos, sendo o diagnóstico definitivo apenas estabelecido ao fim de cerca de 10 anos. 0 doente típico com SHC é um adulto caucasiano (cerca de 80\%), do sexo masculino (rácio homem/mulher 3:27:3) e de idade inferior a 50 anos (a idade média de diagnóstico são os 35 anos) com consumo crónico de canábis. ${ }^{6,10,18} \mathrm{O}$ risco de desenvolver SHC parece depender de vários fatores conhecidos e desconhecidos, incluindo a frequência do consumo de canábis e o método de consumo, assim como eventuais fatores médicos, psiquiátricos, étnicos e condições socioeconómicas. ${ }^{12}$

\section{ETIOPATOGENIA}

A etiopatogenia da SHC encontra-se ainda pouco esclarecida, sendo particularmente desafiante na medida em que os canabinoides são reconhecidos e frequentemente utilizados, nomeadamente em contexto terapêutico, pelos seus efeitos antieméticos e estimuladores do apetite. ${ }^{19,20}$ Alguns dos compostos canabinoides ativos mais comuns incluem o D9-tetra-hidrocanabinol (THC), canabidiol e cannabigerol. Alguns autores hipotetizam que o principal elemento causal da SHC seja o THC. O THC e outros agonistas do recetor canabinoide CB1 têm propriedades antieméticas mediadas pelo agonismo CB1 no hipotálamo que são, contudo, revertidas em determinadas situações. Por outro lado, embora seja antiemético em doses baixas, o canabidiol possui um efeito pró-emético em doses elevadas, que é facilitado pelo cannabigerol. ${ }^{20-23}$ Ao nível do sistema nervoso entérico (SNE), os canabinoides conduzem à diminuição da motilidade gastrointestinal, ao atraso no esvaziamento gástrico e inibem o trânsito através do intestino delgado, primariamente via agonismo CB1. Contudo, em condições normais, estes efeitos são subjugados ao potencial antiemético dos canabinoides no SNC. ${ }^{21,23,24}$
Alguns mecanismos que têm sido propostos para explicar o "paradoxo" da SHC incluem: 1) resultar da exposição cumulativa aos canabinoides; 2) resultar da exposição a canabinoides contaminados com outros produtos; 3) resultar do metabolismo dos canabinoides em compostos pró-eméticos; 3) existir um papel da abstinência de canabinoides; 4) tratar-se de uma reação de tipo idiossincrásico que ocorre em indivíduos vulneráveis e 5) resultar de um desequilíbrio entre os efeitos no SNE e SNC. ${ }^{11,19,22,25}$ Em relação à primeira hipótese, a mais explorada na literatura, o atingimento de níveis tóxicos em consumidores regulares poderá advir do facto dos canabinoides serem compostos lipofílicos, o que Ihes permite acumularem-se no SNC e nos depósitos de gordura periférica. Em situações de lipólise aumentada (i.e., stress ou jejum), pode ocorrer um efeito de "re-intoxicação", independente do consumo atual, atingindo a toxicidade. ${ }^{19}$ Em termos neurobiológicos, e em linha com a hipótese de exposição cumulativa, algumas das explicações aventadas para a génese da SHC consideram que o consumo crónico leva a níveis tóxicos de THC que afetam os recetores CB1 no SNC, envolvidos na termorregulação e na regulação do eixo hipotálamo-pituitária-adrenal (HPA) e sistema nervoso simpático (SNS). Deste forma, estão envolvidos neste processo:

- Recetor CB1: sendo o THC um agonista parcial de CB1, o seu uso crónico e elevadas concentrações podem levar a que tenha ação antagonista (via sub-regulação ou dessensitização dos recetores CB1) e, portanto, pró-emética. Os recetores CB1 presentes na mucosa gástrica podem também ser afetados, produzindo um atraso no esvaziamento gástrico e contribuindo para a ocorrência de vómitos. ${ }^{19,20,26,27}$

- Desregulação do eixo HPA e ativação do SNS: em concentrações fisiológicas normais, os endocanabinoides contribuem para a regulação do eixo HPA, inibindo-o, contribuindo para a homeostase do SNS. Contudo, níveis tóxicos de canabinoides produzem um efeito ativador deste eixo (p.e. ao conduzir a sub-regulação, dessensitização e internalização dos recetores CB1), resultando em menor inibição do eixo HPA e do SNS, gerando respostas hormonais e autonómicas de stress. Esta hipótese é adicionalmente apoiada pelo facto desta síndrome frequentemente ocorrer de manhã (maior ativação do eixo HPA) e pelo efeito terapêutico dos banhos quentes, das benzodiazepinas e antipsicóticos (redução da ativação simpática). ${ }^{27-29}$

Outra possível explicação envolve o recetor vaniloide TRVP1. Este existe no sistema nervoso periférico e central, incluindo no centro de emese, e é ativado por estímulos como elevada temperatura $\left(>43^{\circ} \mathrm{C}\right)$ ou capsaicina. 
O seu agonismo é pró-emético com baixa concentração de ligantes, mas antiemético sob alta concentração de ligantes. Quando estimulado significativamente é dessensibilizado. Nos consumidores crónicos de canabinoides em que ocorre acumulo de substância, pode ocorrer libertação desta com atingimento de concentrações baixas, mas suficientes para produzir efeito pró-emético via estimulação do TRPV1. ${ }^{30,31}$

Igualmente, as explicações do mecanismo subjacente ao benefício dos banhos quentes não geram consenso. As hipóteses propostas incluem: ativação do termorrecetor cutâneo e TRVP1 (banhos quentes levam a dessensibilização do TRVP1, por ativação a temperaturas acima de $43^{\circ} \mathrm{C}$, produzindo efeito antiemético); reversão da hipotermia induzida pelos canabinoides ao nível do hipotálamo; diminuição da ativação do eixo HPA e do SNS; síndrome do "roubo cutâneo" (desvio da circulação sanguínea da circulação esplâncnica para a pele e músculos); regulação de citocinas. ${ }^{30,31}$

Áreas de investigação futura incluem a possibilidade de polimorfismos enzimáticos do citocromo $\mathrm{P} 450$ poderem afetar os níveis de metabolitos pró-eméticos, explicando porque é que apenas alguns dos consumidores são afetados por esta síndrome. ${ }^{24,26}$

\section{COMPLICAÇÕES}

Muitas das complicações da SHC derivam dos banhos de água quente em frequência ou duração excessiva (até 3 a 6 horas). Estes podem resultar em lesões dermatológicas eritematosas e queimaduras pela ação do calor, constituindo também fator de risco para outras complicações, como desidratação e quedas.,12,32 A desidratação, exacerbada pelos vómitos e incapacidade de ingerir líquidos, pode gerar uma depleção de volume significativa, existindo vários casos descritos de lesão renal aguda associada à SHC, de etiologia pré-renal. Estas situações podem assumir uma gravidade extrema, sendo que existem pelo menos dois registos de mortes associadas a estas complicações. ${ }^{33,34}$ Do ponto de vista social, o recurso excessivo a banhos quentes pode gerar tensão junto do agregado que coabita com o doente. ${ }^{32}$

A frequência dos vómitos per se pode, nos casos mais graves, levar a desequilíbrios hidroeletrolíticos, lacerações esofágicas e até mesmo rutura esofágica. Está também descrita a ocorrência de pneumomediastino, associado aos vómitos incoercíveis. ${ }^{35-37}$

Apesar de não se verificar diarreia ou alteração dos hábitos intestinais habituais, a gravidade deste quadro condiciona frequentemente perda ponderal. Na série de Simonetto et al, 83\% reportou perda ponderal, com uma perda média de 14,2 kg. Numa série de casos por
Marillier et al (2017) de 19 pacientes, 12 haviam perdido 5 kg ou mais. 6,38

Embora as complicações cardíacas não pareçam comuns nos quadros de SHC, alguns autores verificaram ocorrência de eventos cardíacos associados. Existe descrição de pelo menos um caso de enfarte agudo do miocárdio, por vasoespasmo, em contexto de SHC. Num outro caso, uma doente de 32 anos desenvolveu miocardiopatia de Takotsubo em contexto de exacerbação da sintomatologia emética. Não é, contudo, estabelecido um elo causal entre ambos. ${ }^{39,40}$

\section{TRATAMENTO}

O tratamento pode ser dividido na terapia da fase hiperemética e na prevenção de recaída. ${ }^{24}$

Os pacientes apresentam-se geralmente no SU na fase hiperemética. Nesta fase deve ter-se em conta a necessidade frequente de vias de administração de terapêuticas alternativas à oral (que pode não ser tolerada) como via endovenosa, sublingual, tópica ou retal. ${ }^{29}$ Inicialmente, aconselha-se fazer reforço da hidratação de forma a compensar as perdas e a dificuldade na ingesta. Os antieméticos tradicionais (p.e metoclopramida) podem ser tentados, embora com provável pouca eficácia. 19,24,41 Alguns autores recomendam tratamento com inibidores da bomba de protões dado os achados de esofagite e gastrite nestes doentes. ${ }^{10,24}$ Alguns casos podem requerer hospitalização devido a dores abdominais severas, depleção de volume, e náuseas e vómitos graves. ${ }^{19,24}$

Os banhos ou duches quentes geralmente são uma terapia standard na $\mathrm{SHC}$, até pelo alívio sintomático que comprovadamente trazem ao doente, sendo o tratamento globalmente mais eficaz. Simonetto et al verificaram que $91 \%$ dos indivíduos melhorava com banhos ou duches quentes, e uma revisão sistemática de Richard et al (2017) ( $n=63$ artigos) corroborou que estes eram quase universalmente eficazes na redução ou ablação dos sintomas. Verifica-se melhoria dos sintomas de náusea e vómitos, dores abdominais e anorexia. $O$ efeito é dependente da temperatura, de início rápido, mas curta duração.9,10,19,24,41

De acordo com a revisão sistemática de Richard et al (2017) mencionada, os tratamentos farmacológicos que aparentam apresentar maior eficácia na melhoria sintomática são:

- Benzodiazepinas, especialmente lorazepam: as propriedades agonistas dos recetores GABA potencialmente explicam os seus efeitos antieméticos ao nível dos núcleos medulares, vestibulares e do trato gastrointestinal; poderá haver contributo do efeito sedativo e da redução da ativação simpática.9,29 
- Haloperidol (relatos de casos a apontar para resolução sintomática após 2 horas e alta 8 horas após administração): ação provavelmente relacionada com bloqueio de recetores dopaminérgicos (D2) pós-sináticos no cérebro, diminuindo o efeito da dopamina na zona de gatilho quimiorrecetora e reduzindo o input ao centro medular de vómito; adicionalmente, possível ação indireta nos recetores CB1.9.22,29,42

- Capsaicina tópica: ação mediada pelo recetor TRPV1 de forma semelhante ao conceptualizado para os banhos quentes; ao ligar com alta afinidade ao TRVP1, a capsaicina pode prejudicar a sinalização nos centros de emese via sobre-estimulação e dessensibilização do TRVP1, funcionando como antiemético.9,22,30,41

De referir que na revisão por Richard et al não foram encontrados estudos prospetivos, aleatorizados e cegos de alta qualidade, sendo que grande parte dos artigos era referente a relatos e séries de casos. ${ }^{\text {? }}$

É feita ainda menção na literatura de possível benefício de: propranolol (por atuação em possível disrupção do SNS); droperidol (Lee et al verificaram benefício em termos de menor permanência hospitalar e menor tempo até alta após a sua administração); olanzapina (aparece indicada como tratamento de SHC em 2 artigos). Os antidepressivos tricíclicos, como amitriptilina, são sugeridos como eficazes no tratamento a longo prazo. Outros fármacos foram referidos como tentados no tratamento da SHC, com menor sucesso (p.e. antihistamínicos, corticosteroides).9,29,43,44

Em termos de prevenção da recaída, o principal objetivo é a cessação do consumo de canabinoides, que leva à regressão dos sintomas de SHC em 1 a 3 meses, sem recrudescimento posterior. Porém, o retorno aos consumos tende a levar a recaída destes sintomas.6,10,13,19 Infelizmente, alguns pacientes mantêm ou aumentam os consumos julgando ser benéfico para a náusea. ${ }^{24}$ É, portanto, essencial informar o doente, esclarecer a relação dos sintomas com os consumos e que a sua cessação leva ao desaparecimento do quadro, e referenciar para apoio especializado quando necessário. Nos casos em que se verifica recusa de abandonar os consumos, o haloperidol tem sido reportado como efetivo a manejar o quadro, tal como os antidepressivos tricíclicos para o tratamento a longo prazo. ${ }^{10,19,45}$

\section{CONCLUSÃO}

A falta de sensibilização para a SHC pode conduzir a testes de diagnóstico invasivos e dispendiosos, inúmeras idas ao SU e manutenção do quadro clínico sem reso- lução sintomática. Aliás, os doentes que sofrem de SHC apresentam frequentemente um longo historial médico, tendo já sido submetidos a vários meios complementares diagnósticos e a várias intervenções terapêuticas. ${ }^{11,12}$ Uma maior consciencialização dos profissionais de saúde sobre a existência da SHC permitirá a identificação dos sintomas e sinais sugestivos deste diagnóstico, possibilitando uma abordagem terapêutica adequada e um maior relato correto dos casos.

A literatura referente à SHC encontra-se em crescimento. Não encontrámos, à data da elaboração deste artigo, dados relativos à realidade na população portuguesa. Algumas questões a abordar no futuro incluem: estudar de forma sistemática a sua prevalência e fatores de risco na população (nomeadamente em Portugal); esclarecer a sua patogénese; otimizar a abordagem terapêutica (p.e. realizar ensaios clínicos aleatorizados); e obter mais dados sobre o seu curso a longo prazo. ${ }^{11,26}$ De referir ainda uma tendência para o aumento do consumo de canabinoides sintéticos, que pode dificultar o diagnóstico; e mudança dos constituintes da canábis consumida, com aumento crescente das variantes de alta potência. ${ }^{21,46}$ Seria relevante perceber empiricamente de que forma estes fatores têm impacto no padrão clínico e epidemiológico da SHC.

\section{RESPONSABILIDADES ÉTICAS}

CONFLITOS DE INTERESSE: Os autores declaram não possuir conflitos de interesse.

SUPORTE FINANCEIRO: O presente trabalho não foi suportado por nenhum subsídio ou bolsa.

PROVENIÊNCIA E REVISÃO POR PARES: Não comissionado; revisão externa por pares.

\section{ETHICAL DISCLOSURES}

CONFLICTS OF INTEREST: The authors have no conflicts of interest to declare.

FINANCIAL SUPPORT: This work has not received any contribution grant or scholarship.

PROVENANCE AND PEER REVIEW: Not commissioned; externally peer reviewed.

\section{REFERÊNCIAS}

1. United Nations Office on Drugs and Crime. World Drug Report. New York: UNODC; 2018.

2. Sahu KK, Mishra A, Naraghi L. Erythema ab igne as a complication of cannabinoid hyperemesis syndrome. BMJ Case Rep. 2019;12:e227836. doi: 10.1136/bcr-2018-227836. 
3. Lee C, Greene SL, Wong A. The utility of droperidol in the treatment of cannabinoid hyperemesis syndrome. Clin Toxicol. 2019;57:773-7. doi: 10.1080/15563650.2018.1564324.

4. Allen JH, Moore GM De, Heddle R, Twartz JC. Cannabinoid hyperemesis: cyclical hyperemesis in association with chronic cannabis abuse. Gut. 2004;53:1566-71.

5. Sontineni SP, Chaudhary S, Sontineni V, Lanspa SJ. Cannabinoid hyperemesis syndrome: Clinical diagnosis of an underrecognised manifestation of chronic cannabis abuse. World J Gastroenterol. 2009;15:1264-6.

6. Simonetto DA, Oxentenko AS, Herman ML, Szostek JH. Cannabinoid hyperemesis: a case series of 98 patients. Mayo Clin Proc. 2012;87:114-9. doi: 10.1016/j.mayocp.2011.10.005.

7. Fleming JE, Lockwood S. Cannabinoid hyperemesis syndrome. Fed Pract. 2017;34:33-6.

8. Aziz I, Palsson OS, Whitehead WE, Sperber AD, Simrén M, Törnblom H. Epidemiology, Clinical characteristics, and associations for rome iv functional nausea and vomiting disorders in adults. Clin Gastroenterol Hepatol. 2019;17:878-86. doi: 10.1016/j.cgh.2018.05.020.

9. Richards JR, Gordon BK, Danielson AR, Moulin AK. Pharmacologic treatment of cannabinoid hyperemesis syndrome: a systematic review. Pharmacotherapy. 2017;37:725-34. doi: 10.1002/phar.1931.

10. Blumentrath C, Dohrman B, Ewald N. Cannabinoid hyperemesis and the cyclic vomiting syndrome in adults: recognition, diagnosis, acute and long-term treatment. Ger Med Sci. 2017;15:Doc06. doi: 10.3205/000247.

11. Sun S, Zimmermann AE. Cannabinoid hyperemesis syndrome. Hosp Pharm. 2013;48:650-5.

12. Wallace EA, Andrews SE, Jelley MJ, Garmany CL. Cannabinoid Hyperemesis syndrome: literature review and proposed diagnosis and treatment algorithm. South Med J. 2011;104:65964.

13. Ruffle JK, Bajgoric S, Samra K, Chandrapalan S, Aziz Q, Farmer AD. Cannabinoid hyperemesis syndrome: an important differential diagnosis of persistent unexplained vomiting. Eur J Gastroenterol Hepatol. 2015;27:1403-8. doi: 10.1097/ MEG.0000000000000489.

14. Habboushe J, Sedor J. Cannabinoid hyperemesis acute renal failure a common sequela of cannabinoid. Am J Emerg Med. 2014;32:690.e1-2.

15. Hernandez JM, Paty J, Price IM. Cannabinoid hyperemesis syndrome presentation to the emergency department: A two-year multicentre retrospective chart review in a major urban area.CJEM. 2017;20:550-5. doi: 10.1017/cem.2017.381.

16. Kim HS, Anderson JD, Sagha O, Heard KJ, Monte AA. Cyclic Vomiting presentations following marijuana liberalization in Colorado. Acad Emerg Med. 2015;22:694-9. doi: 10.1111/ acem.12655.

17. Venkatesan T, Sengupta J, Lodhi A, Schroeder A, Adams K, Hogan WJ, et al. An Internet survey of marijuana and hot shower use in adults with cyclic vomiting syndrome (CVS ). Exp Brain Res. 2014;232:2563-70.

18. Sullivan S. Cannabinoid hyperemesis. Can J Gastroenterol. 2010;24:284-5

19. Beech RA, Sterrett DR, Babiuk J, Fung H. Cannabinoid hyperemesis syndrome: a case report and literature review. J Oral Maxillofac Surg. 2015;73:1907-10. doi: 10.1016/j. joms.2015.03.059.

20. Darmani NA. Cannabinoid-induced hyperemesis: a conundrum-from clinical recognition to basic science mechanisms. Pharmaceuticals. 2010;3:2163-77. doi: 10.3390/ph3072163.
21. Cox B, Chhabra A, Adler M, Simmons J, Randlett D. Case report cannabinoid hyperemesis syndrome: case report of a paradoxical reaction with heavy marijuana use. Case Rep Med. $2012 ; 757696$.

22. Waterson D, Maguire M. Capsaicin topical in emergency department treatment of cannabinoid hyperemesis syndrome. Am J Emerg Med. 2017;35:1977-8. doi: 10.1016/j. ajem.2017.06.038.

23. Goyal H, Singla U, Gupta U, May E. Role of cannabis in digestive disorders. Eur J Gastroenterol Hepatol. 2017;29:135-43. doi: 10.1097/MEG.0000000000000779.

24. Galli JA, Sawaya RA, Friedenberg FK. Cannabinoid Hyperemesis Syndrome. Curr Drug Abus Rev. 2011;4:241-9.

25. Albert K, Sivilotti MLA, Gareri J, Day A, Ruberto AJ, Hookey LC. Hair cannabinoid concentrations in emergency patients with cannabis hyperemesis syndrome. CJEM. 2019;21:47781. doi: $10.1017 /$ cem.2018.479.

26. Brewerton TD, Anderson O. Cannabinoid hyperemesis syndrome masquerading as an eating disorder. Int J Eat Disord. 2016;49:826-9. doi: 10.1002/eat.22515.

27. Schreck B, Wagneur N, Caillet P, Gérardin M, Cholet J, Spadari $M$, et al. Cannabinoid hyperemesis syndrome: Review of the literature and of cases reported to the French addictovigilance network. Drug Alcohol Depend. 2018;182:27-32. doi: 10.1016/j.drugalcdep.2017.09.038.

28. Richards JR. Cannabinoid hyperemesis syndrome: A disorder of the HPA axis and sympathetic nervous system? Med Hypotheses. 2017;103:90-5.

29. Richards JR. Cannabinoid hyperemesis syndrome: Pathophysiology and Treatment in the Emergency Deparment. J Emerg Med. 2017;54:354-63.

30. Dezieck L, Hafez Z, Conicella A, Blohm E, Mark J, Connor $\mathrm{O}$, et al. Resolution of cannabis hyperemesis syndrome with topical capsaicin in the emergency department: a case series. Clin Toxicol. 2017;55:908-13. doi: 10.1080/15563650.2017.1324166.

31. Richards JR, Lapoint JM, Burillo-putze G, Richards JR, Lapoint JM, Burillo-Putze G. Cannabinoid hyperemesis syndrome potential mechanisms for the benefit of capsaicin and hot water hydrotherapy in treatment. Clin Toxicol. 2018;56:15-24. doi: 10.1080/15563650.2017.1349910.

32. Hinton KL, Chui JS, Mcwhorter KA, Jallad RH, Siple JF. Cannabinoid hyperemesis syndrome: a paradoxical case. Ann Pharmacother. 2016;50:1071-2. doi: 10.1177/1060028016660719.

33. Klassen J, Wilson G. Cannabinoid hyperemesis syndrome masquerading as uremia: an educational case report. Can J Kidney Heal Dis. 2018;5. doi: 10.1177/2054358118791146.

34. Nourbakhsh M, Miller A, Gofton J. Cannabinoid hyperemesis syndrome: reports of fatal cases. J Forensic Sci. 2018;64:270-4.

35. Morris R, Fisher M. Cannabinoid hyperemesis syndrome: a specific cause of cyclical vomiting. Int J Adolesc Med Heal. 2014;26:153-6.

36. Nicolson SE, Denysenko L, Mulcare JL, Vito JP, Chabon B. Cannabinoid hyperemesis syndrome: a case series and review of previous reports. Psychosomatics. 2012;53:212-9. doi: 10.1016/j.psym.2012.01.003

37. Swanson M, Epperly T. Vomiting, abdominal pain, compulsive bathing--Dx? J Fam Pract. 2014;63:257-9.

38. Marillier M, Batisse A, Edel Y, Nguyen A, Chevallier C, Pfau $\mathrm{G}$, et al. Cannabinoid hyperemesis syndrome $(\mathrm{CHS})$ a parisian case series. J Clin Psychopharmacol. 2017;37:739-43. doi: 10.1097/JCP.0000000000000791. 
39. Pierard S, Hantson P. Coronary vasospasm complicating cannabinoid hyperemesis syndrome. J Cardiol Cases. 2017;15:1158. doi: 10.1016/j.jccase.2016.12.001.

40. Nogi M, Fergusson D, Chiaco J. Mid-ventricular variant Takotsubo cardiomyopathy associated with cannabinoid hyperemesis syndrome: a case report. Hawaii J Med Public Heal. 2014;73:115-8.

41. Khattar N, Routsolias JC. Emergency department treatment of cannabinoid hyperemesis syndrome: a review. Am J Ther. 2018;25:e357-61. doi: 10.1097/MJT.0000000000000655.

42. Hickey JL, Pharmd JCW, Mycyk MB. Haloperidol for treatment of cannabinoid hyperemesis syndrome. Am J Emerg Med. 2013;31:1003.e5-1003.e6.

43. Lee C, Greene SL, Wong A. The utility of droperidol in the treatment of cannabinoid hyperemesis syndrome. Clin Toxicol. 2019;57:773-7. doi: 10.1080/15563650.2018.1564324.

44. Richards J, Dutczak O. Propranolol treatment of cannabinoid hyperemesis syndrome a case report. J Clin Psychopharmacol. 2017;37:482-4. doi: 10.1097/JCP.0000000000000735.

45. Lapoint J, Meyer S, Yu CK, Koenig KL. Cannabinoid hyperemesis syndrome: public health implications and a novel model treatment guideline. West J Emerg Med. 2018;19:380-6. doi: 10.5811/westjem.2017.11.36368.

46. Bick B, Szostek J, Mangan T. Synthetic cannabinoid leading to cannabinoid hyperemesis syndrome. Mayo Clin Proc. 2014;89:1168-9. doi: 10.1016/j.mayocp.2014.06.013. 